\title{
VRCircuit: Realidade Virtual aplicada ao Ensino de Circuitos Elétricos
}

\author{
Pablo de L. Sanches ${ }^{1}$, Leonardo M. Faêda ${ }^{1}$, Alex F. V. Machado ${ }^{1}$ \\ ${ }^{1}$ Departamento Acadêmico de Ciência da Computação \\ Instituto Federal de Educação, Ciência e Tecnologia do Sudeste de Minas Gerais \\ Campus Rio Pomba, Minas Gerais, Brasil \\ \{pablo.lara.sanches, leonardo.faeda\}@gmail.com, alexcataguases@hotmail.com
}

\begin{abstract}
One of the main questions in the teaching research field is the utilization of technology to help the learning in the classroom. The fast technological development made this an indispensable fact, encouraging the development of educational applications in various disciplines. Educational games that respects certain characteristics and requirements can become useful tools to support the teachers work in the classroom. This project shows the development, experiments and tests of the game VRCircuit, geared towards teaching of physics in higher education. With the help of a virtual reality equipment, we propose an application to help the teaching of electric circuits geared towards undergraduate students in Computer Science or related fields.
\end{abstract}

Resumo. Uma das principais questões atuais de pesquisa em ensino é a utilização da tecnologia no auxílio da aprendizagem em sala de aula. O veloz desenvolvimento tecnológico tornou esse fato imprescindivel, incentivando o desenvolvimento de aplicações educacionais em diversas disciplinas. Os jogos educacionais respeitando certas características e requisitos, podem ser instrumentos úteis de apoio ao trabalho dos professores na sala de aula. Este projeto apresenta o desenvolvimento, experimentos e testes do jogo VRCircuit, voltado para o ensino de física na educação superior. Com o auxílio do óculos de realidade virtual, propomos uma aplicação para o reforço do conteúdo de circuitos elétricos voltado ao aluno da graduação em Computação ou áreas afins.

\section{Introdução}

Há várias linhas pedagógicas que defendem seus métodos para o avanço do educando. Atualmente, a maioria delas está atenta ao fato da introdução da tecnologia como auxílio em seus processos pedagógicos [Zorzal et al. 2006].

Os últimos anos têm sido marcados por uma aceleração no processo de desenvolvimento dos recursos midiáticos na metodologia de aprendizagem. Acredita-se que a ciência das tecnologias disponíveis na sociedade, em especial os jogos, é fundamental para o trabalho educacional transformador e de qualidade, e.g. [Leite 1996].

O jogo pode ser considerado como um importante meio educacional, pois propicia um desenvolvimento integral e dinâmico nas áreas cognitiva, afetiva, linguística, social, moral e motora. Podendo contribuir para a construção da autonomia, criatividade, responsabilidade e cooperação das crianças e adolescentes, desde que sejam planejados e 
VI Congresso Brasileiro de Informática na Educação (CBIE 2017)

Anais do XXVIII Simpósio Brasileiro de Informática na Educação (SBIE 2017)

trabalhados de uma forma crítica, que possibilite a aprendizagem de uma maneira significativa ao aprendiz.

Segundo [Calisto et al. 2010], os jogos educativos fornecem uma importante contribuição à aquisição de conhecimento, pois são ambientes capazes de disseminar informações. De fato, um jogo educativo estimulará a atenção dos alunos, ao mesmo tempo em que os motivará através de atividades de competição e cooperação.

Segundo [Fiolhais and Trindade 2003], uma das particularidades das disciplinas das Ciências em geral, que as tornam difíceis de aprendizagem para os alunos, é o fato de abordarem conceitos abstratos em uma ampla medida não intuitiva. A capacidade de absorção de informação dos estudantes é reduzida. Em decorrência, muitos não conseguem entender a ligação destas com a vida real. Esta dificuldade de percepção é tratada em partes por métodos de experimentação através de atividades laboratoriais. Essa forma de ensino vai ao encontro à observação sobre a ligação entre os dois modos de aprender como método de maximizar o aprendizado.

Observando as naturezas de determinadas disciplinas, no caso da física, conseguimos perceber que ela é uma das áreas do conhecimento que mais se proporciona o aprendizado por experimentos e observações de fenômenos, e.g. [Pinho 1996]. Por tal fato, explica-se o aumento de aplicações e métodos desenvolvidos para relacionar o ensino prático aos tópicos desta matérias.

Uma tentativa que abrange essa situação é utilizar da tecnologia como forma de simulação de experiências. Nesta metodologia de uso de dispositivos para apoiar a educação, a tecnologia realiza uma ação de facilitador, na medida em que possibilita ao educador o acesso a aplicações antes indisponíveis. No entanto, sem ser o centro do procedimento pedagógico e nem ser o único responsável pelo êxito da aprendizagem.

Para que os alunos possam ter interesse pelo aprendizado, ele deve ser feito de maneira espontânea, mas para que esse interesse seja despertado é necessário que a forma de estudar seja prazerosa. No entanto, o que observamos é que a educação caminha na contramão da satisfação e da espontaneidade. Na maioria das escolas é ensinado aos alunos que sala de aula não é lugar para brincadeira, tornando a aprendizagem maçante e desprazerosa.

Segundo [Ramos and Ferreira 1990], a aprendizagem para poder ser absorvida tem que partir de um processo interior do sujeito. Sabe-se que jogos e brincadeiras são fontes provenientes de atração, tornado essas atividades voluntárias do ser humano. Os jogos proporcionam ambientes divertidos, incluindo a exploração dos benefícios dos jogos educacionais, em função de suas características lúdicas, de envolvimento, desenvolvimento, habilidades e de construção do conhecimento [Forte and Kirner 2009].

Desta maneira, foi na forma lúdica dos jogos que buscamos condições favoráveis para desenvolver conteúdos de física. Portanto este trabalho apresenta o desenvolvimento de um jogo educativo para o ensino de circuitos elétricos em ambiente de realidade virtual, para justificar as vantagens ressaltadas do potencial de utilização dos jogos educacionais.

\subsection{A realidade virtual no atual mercado de jogos}

A realidade virtual voltada para o mercado de jogos começou em 1995, quando a empresa de jogos, Nintendo, lançou um videogame portátil chamado Virtual Boy. Este 
VI Congresso Brasileiro de Informática na Educação (CBIE 2017)

Anais do XXVIII Simpósio Brasileiro de Informática na Educação (SBIE 2017)

videogame não atingiu sucesso de mercado, pois os usuários reclamavam de dor de cabeça, além de ser um console caro e com um efeito 3D de má qualidade para a época [Zachara and Zagal 2009].

Em 2012, a empresa Oculus abriu uma campanha kickstarter para lançar um dispositivo de realidade virtual que viria a revolucionar este mercado. O Oculus Rift, como foi chamado o produto, teve o seu primeiro lançamento em 2013, com uma versão de desenvolvedor [Boas 2013]. A qualidade do Rift proporcionou uma imersão em jogos nunca vista em nenhum outro dispositivo lançado para o mercado antes. A partir deste lançamento, diversos produtores puderam criar jogos e aplicativos usando realidade virtual, e isto gerou um crescimento enorme desta tecnologia.

Franquias de jogos já consolidados no mercado dos videogames começaram a migrar para esta nova tecnologia. A série de jogos Resident Evil, que já é famosa por seus diversos jogos de terror/suspense, resolveu inovar com o jogo Resident Evil 7, o primeiro da franquia a utilizar um dispositivo de realidade virtual.

O jogo VR-Engage [Virvou et al. 2002] usa a realidade virtual na educação, através do ensino de geografia aos estudantes de uma maneira divertida e atrativa. $\mathrm{O}$ jogador avança pelo jogo respondendo perguntas de geografia aos dragões, que guardam as portas que necessitam ser atravessadas para se chegar ao livro da sabedoria.

\section{Trabalhos relacionados}

Existem trabalhos com o intuito de melhorar o ensino-aprendizagem, principalmente, em disciplinas voltadas para Programação, Ciências Exata e Robótica, muitos desses trabalhos são desenvolvidos para computadores e celulares, mas a realidade virtual ainda está pouco presente em jogos com foco na educação de disciplinas escolares.

O EletriCidade é uma proposta não implementada de um jogo educacional, com o objetivo de ensinar conceitos fundamentais de eletricidade voltado principalmente para alunos do ensino médio. Ele é organizado em fases onde o personagem principal possui várias missões para cumprir. A cada fase cumprida com sucesso, o jogador é contemplado com pontos e acessórios de que vai precisar posteriormente. No enredo, uma série de fenômenos malucos, ocasionados pela má utilização dos recursos naturais na exploração da energia, causa caos e destruição em uma cidade [Hornes et al. 2009].

[Silva and de Sousa 2013] propõem um jogo educativo para o ensino de Física Quântica com o auxílio da realidade virtual. O jogo possui como principal característica o uso da capacidade de furtividade. Nele o jogador deve evitar ser percebido enquanto coleta informações, responde às questões para encontrar as partículas, e anda pelo percurso do cenário. Quando o jogador consegue encontrar uma partícula, ela é inserida no inventário, permitindo abrir a porta para sair da fase e iniciar a próxima.

No trabalho de [Forte and Kirner 2009] aborda uma discussão sobre o uso de tecnologias na educação e apresenta uma proposta de software, chamado de ARTutor, que tem como objetivo a resolução de desafios e estudos teóricos relacionados à matemática ou física, baseado em realidade aumentada.

O principal diferencial do presente trabalho em relação aos demais surge da utilização do jogo como uma ferramenta para atrair a atenção de alunos e incentivá-lo 
VI Congresso Brasileiro de Informática na Educação (CBIE 2017)

Anais do XXVIII Simpósio Brasileiro de Informática na Educação (SBIE 2017)

na busca do conhecimento, através de circuitos elétricos em um ambiente de realidade virtual.

\section{Metodologia de desenvolvimento}

Para auxiliar o desenvolvimento do jogo VRCircuit foi escolhido a metodologia ágil Scrum. Essa técnica foi adotada pelo o sucesso de muitos desenvolvedores de jogos eletrônicos que a utilizaram. O projeto seguiu alguns dos princípios propostos por este processo como representado na Figura 1, que foram seguidos e adequados para o desenvolvimento do projeto, com forma de orientação e cumprimento dos prazos estabelecidos para cada fase do processo de desenvolvimento.

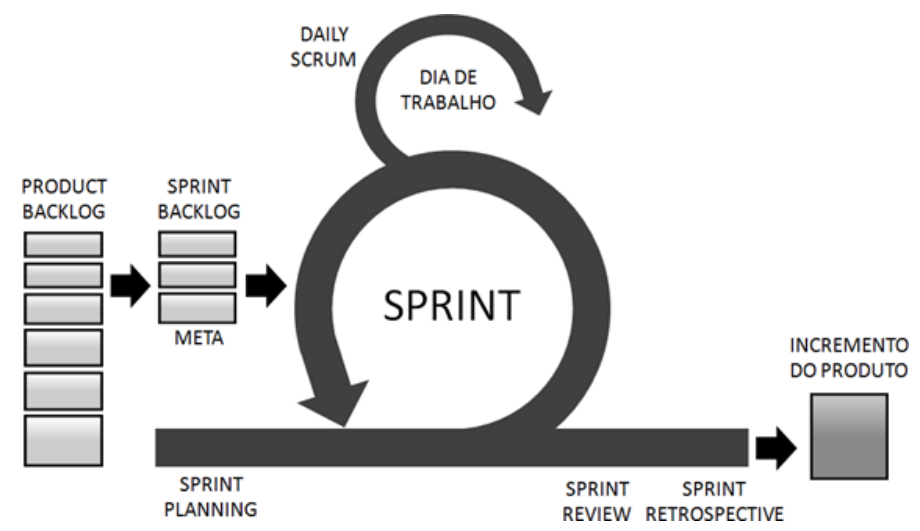

Figura 1. Metodologia Scrum [Otávio 2016].

No método Scrum, os projetos são divididos em sprints que representam um tempo definido dentro do qual um conjunto de atividades devem ser executadas. O tempo proposto para esse projeto foi de 90 dias. No início dele foi realizada uma sprint planning, que é uma reunião com todos membros do grupo para o planejamento das atividades e a montagem de uma estrutura para o jogo descrito no fluxograma (Figura 2). Onde ficou decidido dividir o jogo em duas partes, uma onde o jogador poderá consultar e estudar as fórmulas de física e outra no qual o jogador colocará em prática o conteúdo adquirido na forma de jogo.

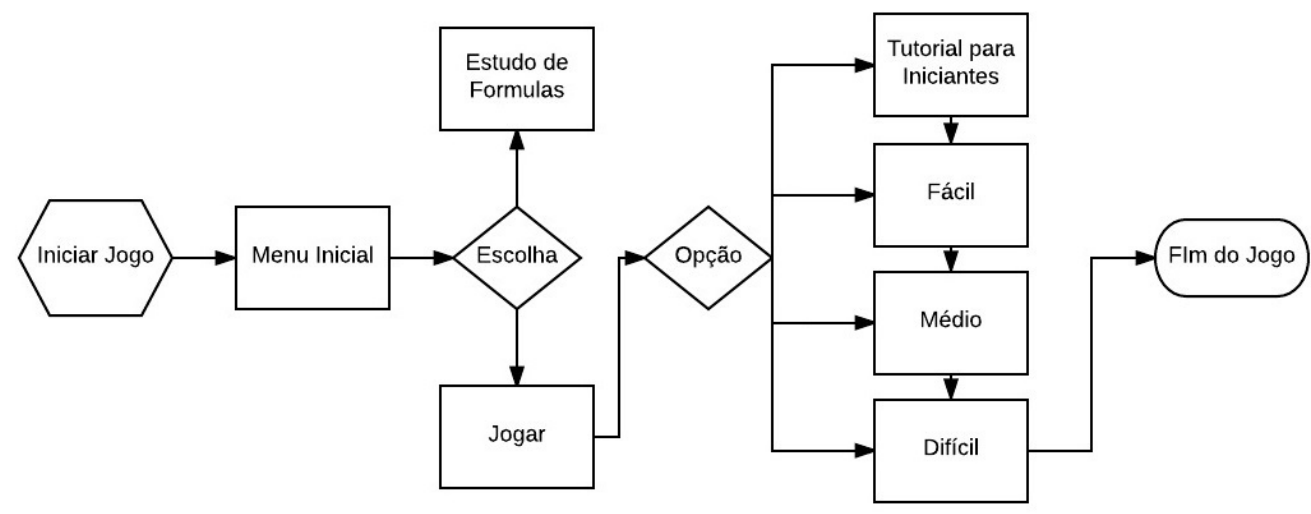

Figura 2. Fluxograma do jogo.

A metodologia utilizada na construção, avaliação e testes do jogo foi composta por 10 etapas: (1) Identificação e estudo do problema; (2) Organização da solução; (3) 
VI Congresso Brasileiro de Informática na Educação (CBIE 2017)

Anais do XXVIII Simpósio Brasileiro de Informática na Educação (SBIE 2017)

Definição do escopo do jogo; (4) Desenvolvimento do jogo; (5) Testes do jogo; (6) Elaboração e aplicação de avaliação do jogo na escola; (7) Aplicação do jogo na escola; (8) Aplicação da avaliação diagnóstica; (9) Análise dos resultados; (10) Organização e publicação dos resultados.

\section{Proposta do VRCircuit}

Nesta seção são relatadas as funcionalidades do jogo, abordando sua mecânica, estrutura, interface e jogabilidade. Além disso, é demonstrado as ferramentas utilizadas para a sua construção, equipamentos necessários para estar jogando e como foi realizada a construção do seu ambiente virtual.

A principal ideia para a concepção do VRCircuit é uma abordagem lúdica, diferente do ensino tradicional da física elétrica, através de um jogo com a mecânica de um puzzle 3D com navegação em primeira pessoa ,em que o usuário possa interagir com um ambiente virtual, tornando a aprendizagem mais fácil e divertida para o aluno.

\subsection{Ferramentas e equipamentos}

Para a criação do projeto foi utilizado o programa Unity 3D na versão 5.5.2, que é uma plataforma dedicada para a criação de jogos. Nela foram desenvolvidas as cenas e a mecânica. Os jogos desenvolvidos nesta ferramenta podem funcionar em diversas plataformas, em nossa abordagem foi escolhido para desktop e dispositivos móveis. Alguns objetos presentes nos cenários foram criados usando o Blender, que é uma ferramenta de modelagem em 3D.

Os equipamentos necessários para estar jogando na versão desktop é um computador, juntamente com um óculos de realidade virtual, podendo ser controlado pelo teclado e mouse ou por um controle. Para jogar em dispositivos móveis é necessário um cardboard junto com o smartphone e um controle sem fio.

\subsection{Especificação do jogo}

A figura 3(a) mostra a tela inicial do jogo, onde existem as opções de Iniciar, Estudar, Informações e Sair. Ao selecionar o botão jogar, uma tela com as opções de dificuldade é apresentada ao jogador, como mostrado na figura 3(c). Ao selecionar o botão de Estudar, o jogador passa para a tela seguinte, como mostrado na figura 3(b), onde o jogador pode revisar alguns dos conceitos físicos utilizados no VRCircuit. Ao selecionar o botão de Informações, as informações sobre os desenvolvedores do jogo são mostradas na tela. Ao selecionar a opção Sair, a aplicação fecha.

O jogo foi construído com a perspectiva do usuário na primeira pessoa, onde a câmera está na visão do personagem. Este modo de câmera foi escolhido por tratar de um jogo em realidade virtual, proporcionando ao usuário uma maior imersão no ambiente, tornando assim uma experiência mais realista.

O jogo começa com o personagem em um cenário, que muda dependendo da dificuldade escolhida pelo jogador. Cada cena possui uma proposta de circuito elétrico, podendo conter resistências em série, paralelo ou mista, conjunto de baterias e uma determinada corrente. Ao iniciar a fase o circuito elétrico proposto apresenta problemas que devem ser corrigidos pelo jogador, utilizando os conceitos de física previamente adquiridos em sala de aula ou pelo jogo. 


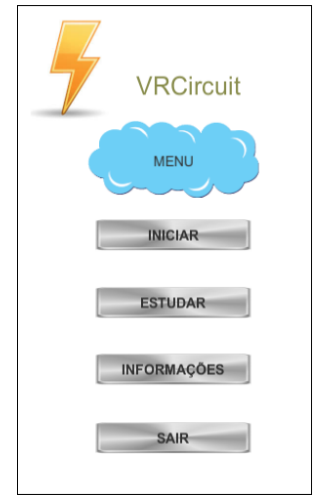

(a) Menu do jogo

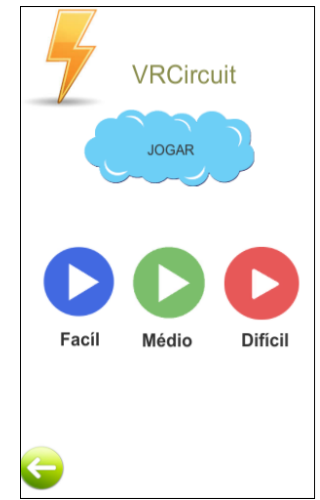

(b) Fases do jogo

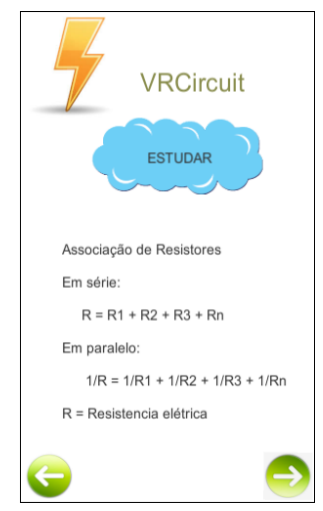

(c) Conceitos de física usados no jogo

Figura 3. Imagens do Jogo

O jogador controla seu personagem usando o teclado e mouse ou um controle, podendo movimentar e rotacionar pelo cenário, consultar a todo instante materiais com conceitos físicos e interagir com os objetos da cena. Todos instrumentos que possuem alguma interação tem a cor azul para facilitar a comunicação do usuário com o jogo e uma descrição que aparece quando o jogador aproxima, informando sua função e especificação (Figura 4).

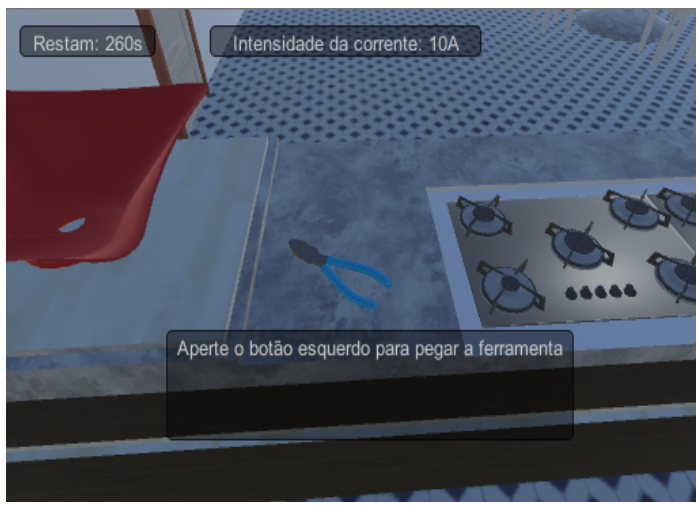

(a) Ferramenta

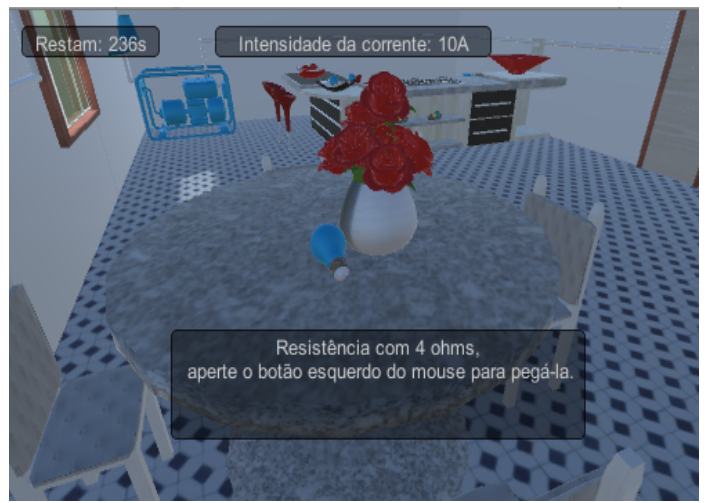

(b) Resistência

Figura 4. Objetos utilizados no jogo

Para o jogador resolver o problema apresentado pelo circuito elétrico, ele deve observar qual tipo de circuito está sendo apresentado pelo cenário, a voltagem da bateria disponível, quais problemas estão fazendo com que o circuito não funcione, o tempo disponível para a conclusão desta tarefa e a intensidade da corrente. O tempo e a intensidade são informados no canto superior esquerdo do jogo. Então, é preciso procurar por ferramentas (Figura 4(a)) e resistências (Figura 4(b)) que resolvam os problemas.

O jogador terminando de montar o circuito elétrico utilizando de seus conhecimentos na prática, precisa ligar a bateria para ver se sua escolhas foram corretas. Ligando a bateria as luzes se acenderá com uma animação da corrente elétrica passando pelos fios e a mensagem de "Parabéns!" aparece na tela (Figura 5(a)), caso o participante tenha corrigido os problemas corretamente. Mas se as escolhas forem erradas, a bateria soltará 
VI Congresso Brasileiro de Informática na Educação (CBIE 2017)

Anais do XXVIII Simpósio Brasileiro de Informática na Educação (SBIE 2017)

faíscas, e a mensagem de "tente novamente" aparece na tela, como mostrado na figura $5(\mathrm{~b})$.

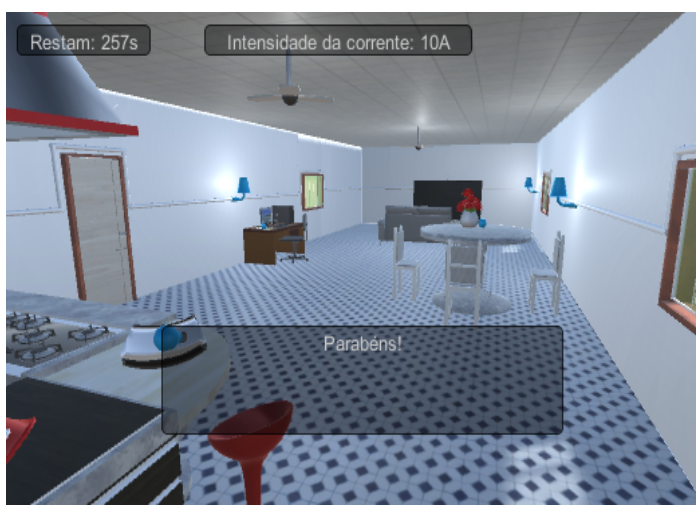

(a) Circuito concluído com sucesso

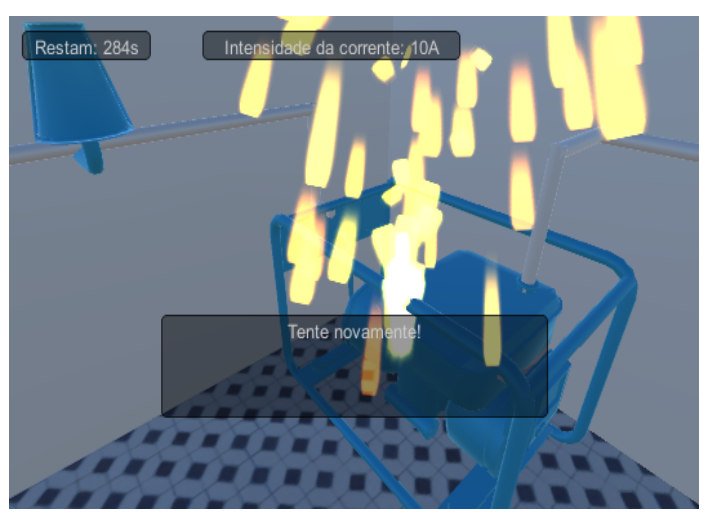

(b) Circuito concluído com erros

Figura 5. Imagens do Jogo

\section{Experimentos}

Para [Silva 2009] o professor ao escolher um software educacional adequado, precisa ter como critérios: o conceito a ser ensinado, o conhecimento sobre a facilidade de uso com os alunos, o seu manuseio pessoal, para após avaliar e planejar uma situação de ensino. A avaliação de software educacional deve incluir aspectos como usabilidade, a eficácia das ferramentas de auxílio, além da aprendizagem alcançada. Elas enfatizam a necessidade de utilizar tanto a avaliação orientada para o produto quanto a avaliação orientada para o usuário.

Como forma de verificar se o jogo está pronto para o uso, sem que o aplicativo apresente algum erro de execução e que seu verdadeiro propósito em ser um jogo educativo e lúdico estava sendo executado. Foram realizados testes e discussões com o objetivo de descobrir problemas e validar o jogo, através das críticas e relatos dos participantes podemos descobrir e resolver os impasses observados.

A avaliação deste jogo foi realizada segundo os critérios de [Medeiros and Schimiguel 2012]:

- Qualidade do conteúdo - Veracidade e apresentação equilibrada das ideias com nível apropriado de detalhes, enfatizando os pontos significantes;

- Alinhamento do Objetivo da Aprendizagem - Alinhamento entre as metas de aprendizagem, atividades, avaliações e características dos alunos;

- Motivação - Capacidade de motivar o interesse do jogador;

- Imersão - Capacidade de envolver o jogador profundamente;

- Objetivos Claros - Metas claras do que deve ser realizado;

- Feedback e Adaptação - Elaboração do feedback positivo e negativo do jogo. Conteúdo que se adapta de acordo com a habilidade do jogador;

- Apresentação - Informação visual; 
VI Congresso Brasileiro de Informática na Educação (CBIE 2017)

Anais do XXVIII Simpósio Brasileiro de Informática na Educação (SBIE 2017)

- Interação Social - Dispor meios de interação com outros jogadores; e

- Reusabilidade - Capacidade de ser utilizado em diferentes contextos de aprendizagem e com alunos de diferentes idades e interesses.

[Godoi and Padovani 2015] observaram que instrumentos avaliativos de software educacional podem ser classificados em: checklists, diretrizes, escalas de avaliação, formulários, modelo conceitual, questionários e sistemas ou de forma híbrida. Dentre os instrumentos avaliativos, foi escolhido o questionário de avaliação para verificar o impacto do jogo no processo de ensino-aprendizagem da física.

\subsection{Testes}

Os testes foram aplicados no [omitido para revisão], em alunos do terceiro período do bacharelado em Ciência da Computação que estão cursando a disciplina de Física Elétrica, totalizando 10 indivíduos com idade entre 18 e 24 anos.

Para conseguir respostas aos questionamentos, ao final do jogo, foi aplicado um questionário individualmente para cada aluno. Para a análise dos resultados foi utilizado o método de frequência relativa em todas as questões aplicadas aos participantes. O qual apresentou perguntas assertivas e o aluno tinha que escolher as opções de: Discordo Totalmente (DT), Discordo Parcialmente (DP), Indiferente (IN), Concordo Parcialmente (CP) e Concordo Totalmente (CT). As questões estão apresentadas na Tabela 1.

Tabela 1. Principais resultados da avaliação.

\begin{tabular}{|l|c|c|c|c|c|}
\hline \multicolumn{1}{|c|}{ Perguntas } & DT & DP & IN & CP & CT \\
\hline $\begin{array}{l}\text { Entendi rapidamente o que deveria fazer para inte- } \\
\text { ragir com o jogo. }\end{array}$ & $0 \%$ & $10 \%$ & $10 \%$ & $0 \%$ & $80 \%$ \\
\hline $\begin{array}{l}\text { O jogo reconheceu corretamente meus movimen- } \\
\text { tos no cenário. }\end{array}$ & $0 \%$ & $0 \%$ & $0 \%$ & $10 \%$ & $90 \%$ \\
\hline Os objetos estavam adequados. & $0 \%$ & $0 \%$ & $10 \%$ & $20 \%$ & $70 \%$ \\
\hline Compreendi facilmente as instruções. & $10 \%$ & $0 \%$ & $0 \%$ & $10 \%$ & $80 \%$ \\
\hline Identifiquei assuntos dados em sala de aula. & $0 \%$ & $0 \%$ & $10 \%$ & $0 \%$ & $90 \%$ \\
\hline Achei que faltaram explicações nas instruções. & $60 \%$ & $30 \%$ & $0 \%$ & $10 \%$ & $0 \%$ \\
\hline $\begin{array}{l}\text { Senti-me motivado para avançar pelos fases pro- } \\
\text { postas. }\end{array}$ & $0 \%$ & $0 \%$ & $0 \%$ & $20 \%$ & $80 \%$ \\
\hline $\begin{array}{l}\text { A utilização da realidade virtual me estimulou a } \\
\text { continuar jogando. }\end{array}$ & $0 \%$ & $0 \%$ & $0 \%$ & $0 \%$ & $100 \%$ \\
\hline Você conseguiu sentir imerso em um cenário. & $0 \%$ & $0 \%$ & $20 \%$ & $0 \%$ & $80 \%$ \\
\hline O jogo foi atrativo e interessante. & $0 \%$ & $0 \%$ & $0 \%$ & $10 \%$ & $90 \%$ \\
\hline
\end{tabular}

Os resultados obtidos pela aplicação dos questionários apontam que a maioria dos participantes respondeu de forma positiva sobre a usabilidade. Pois, $100 \%$ deles qualificaram que o jogo reconheceu corretamente seus movimentos no cenário, enquanto outros $80 \%$ concordaram com a afirmativa: "entendi rapidamente o que deveria fazer para interagir com o jogo", apontou a satisfação ao interagir com a aplicação. 
VI Congresso Brasileiro de Informática na Educação (CBIE 2017)

Anais do XXVIII Simpósio Brasileiro de Informática na Educação (SBIE 2017)

A maioria dos alunos $90 \%$ concordaram que conseguiram identificar os conteúdos trabalhados em sala de aula pelos professores no jogo. Este resultado é importante, pois reflete que o aluno conseguiu entender os conteúdos trabalhados na sala de aula e usálos sob uma perspectiva diferente. Outro resultado que desperta atenção é a motivação dos alunos, já que todos concordaram que se sentiram motivados à medida que foram avançando pelos estágios.

Os resultados também apontaram que a maioria dos alunos aprovou a utilização da realidade virtual e conseguiu sentir-se imersos no ambiente de jogo, fazendo com que ele fique mais atrativo e interessante de ser jogado.

\section{Conclusão e Trabalhos Futuros}

O mercado de jogos eletrônicos é um meio em expansão. Sendo assim, os jogos permeiam cada vez mais o dia a dia de grande parte da população. Assim como o cinema, a televisão e a Internet, é inevitável que haja aplicações focadas no ensino, mas o sucesso das mesmas depende de um fator diferencial, que os jogos sejam lúdicos, onde os jogadores possam aprender e ao mesmo tempo se divertir.

A principal contribuição deste trabalho foi utilizar de recursos tecnológicos por meio de um jogo digital para auxiliar os alunos no aprendizado de física, que possuem dificuldades na absorção deste conteúdo. Com esta abordagem construtivista, os alunos trabalham conceitos aprendidos em sala de aula sob uma perspectiva diferente, uma vez que eles estão acostumados com problemas rotineiros apresentados pelo professor e abordagens que fogem do comum acabam despertando maiores interesses nos alunos.

Após testes realizados com alunos jogando o VRCircuit, foi verificado que o mesmo pode vir a colaborar fortemente no processo de ensino/aprendizagem visto que $100 \%$ dos estudantes o avaliaram positivamente. Em atualizações futuras, serão adicionadas fases que abrangem mais conceitos de física, não apenas física elétrica.

\section{Referências}

Boas, Y. (2013). Overview of virtual reality technologies. In Interactive Multimedia Conference 2013.

Calisto, A., Barbosa, D., and Silva, C. (2010). Uma análise comparativa entre jogos educativos visando a criação de um jogo para educação ambiental. In Brazilian Symposium on Computers in Education (Simpósio Brasileiro de Informática na Educação-SBIE), volume 1.

Fiolhais, C. and Trindade, J. (2003). Fisica no computador: o computador como uma ferramenta no ensino e na aprendizagem das ciências fısicas. Revista Brasileira de Ensino de Fisica, 25(3):259-272.

Forte, C. E. and Kirner, C. (2009). Usando realidade aumentada no desenvolvimento de ferramenta para aprendizagem de física e matemática. In $6^{\circ}$ Workshop de Realidade Virtual e Aumentada-WRVA, volume 2009, pages 1-6.

Godoi, K. A. and Padovani, S. (2015). Instrumentos avaliativos de software educativo: uma investigação de sua utilização por professores. Estudos em Design, 19(1).

Hornes, A., Grachinski, L., da Silva, S. d. C. R., and Koscianski, A. (2009). Os jogos computacionais no ensino de física. ENPEC, VII. 
VI Congresso Brasileiro de Informática na Educação (CBIE 2017)

Anais do XXVIII Simpósio Brasileiro de Informática na Educação (SBIE 2017)

Leite, L. S. (1996). Tecnologia educacional: descubra suas possibilidades na sala da aula. Diadorim Editora.

Medeiros, M. and Schimiguel, J. (2012). Uma abordagem para avaliação de jogos educativos: ênfase no ensino fundamental. In Brazilian Symposium on Computers in Education (Simpósio Brasileiro de Informática na Educação-SBIE), volume 23.

Pinho, M. S. (1996). Realidade virtual como ferramenta de informática na educação. Anais do SBIE (Simpósio Brasileiro de Informática na Educação), Belo Horizonte, Minas Gerais.

Ramos, E. d. F. and Ferreira, N. C. (1990). Brinquedos e jogos no ensino de física. São Paulo.

Silva, G. C. and de Sousa, P. M. (2013). O uso da realidade virtual para o ensino de física quântica use of virtual reality in learning of quantum physics. In Anais dos Workshops do Congresso Brasileiro de Informática na Educação, volume 2.

Silva, R. (2009). Avaliação de software educacional: critérios para definição da qualidade do produto. III Simpósio Nacional ABCiber. Brasil: ESPM/SP-Campus Francisco Gracioso.

Virvou, M., Manos, C., Katsionis, G., and Tourtoglou, K. (2002). Vr-engage: A virtual reality educational game that incorporates intelligence. In Proceedings of IEEE international conference on advanced learning technologies, pages 16-19.

Zachara, M. and Zagal, J. P. (2009). Challenges for success in stereo gaming: a virtual boy case study. In Proceedings of the international conference on Advances in Computer Enterntainment Technology, pages 99-106. ACM.

Zorzal, E. R., Cardoso, A., Kirner, C., and Lamounier, E. (2006). Realidade aumentada aplicada em jogos educacionais. In $V$ Workshop de Educação em Computação e Informática do Estado de Minas Gerais-WEIMIG. 